\title{
Excimer Laser Photoablation with in-situ Masking for Fabrication of Stretchable Pressure Sensor Arrays
}

\author{
Kevin L. Lin and Kanti Jain \\ Electrical and Computer Engineering \\ University of Illinois at Urbana-Champaign \\ Urbana, Illinois, USA \\ kllin2@illinois.edu
}

\begin{abstract}
Stretchable sensor arrays have applications in robotics, structural health monitoring (SHM) devices, and electronic textiles applications where they can be mounted directly on nonplanar surfaces. For robotics, pressure sensor arrays mimic the human sense of touch, providing sensory feedback to help the robot move in a controlled manner. Our research investigates a novel microelectronic process sequence for fabricating stretchable capacitive pressure sensors on polymer substrates, where metal patterns serve both as functional electrode layers and as in-situ masks for excimer laser photoablation. This approach reduces the number of photomasks and processing steps, is scalable for large-area arrays, and is adaptable for a variety of materials and designs. Individual $200 \mu \mathrm{m}$-sized sensors show a capacitance change of $60 \mathrm{fF}$ with an applied pressure of $500 \mathrm{kPa}$. Pressure sensors are fabricated as a $4 \times 4$ array connected with redundant stretchable interconnects to demonstrate successful readout of different pressure profiles.
\end{abstract}

\section{INTRODUCTION}

As the field of robotics advances, it becomes more important for the robot to have tactile feedback, such that it can mimic the human sense of touch. When manipulating small or fragile objects, tactile feedback allows the robot to know how much pressure it is applying on the object to avoid damage. Similarly, tactile feedback enables the robotic hand to interact with humans in a friendly and controlled manner. The need for tactile feedback can be realized by an "electronic skin" MEMS pressure sensor array that is flexible, and conformable. The conformability improves robustness and enables the sensor to be mounted on non-planar surfaces such as a robotic hand. In addition to tactile sensors for robotics, stretchable capacitive pressure sensor arrays also have applications in the biomedical, aerospace, and automotive industries [1-3].

There are several approaches to fabricating flexible pressure sensor arrays. One approach uses a polymer substrate with a conductive rubber mesh and organic thin-film transistors (TFTs) such that the change in pressure is transduced into a change in resistance of the conductive rubber layers [2-3]. To improve stretchability and conformability, selective parts of the polymer substrate are removed to form a sensor array mesh. Another approach utilizes strain sensor arrays, fabricated by selectively adding spin-on polymer layers and thin-films of metal onto a polyimide substrate. A change in pressure is transduced into a strain and a corresponding change in resistance [4]. A third approach uses a flexible stainless steel substrate, polyimide layers and two layers of metal such that a change in pressure is transduced into a change in capacitance for each element in the array [1]. In general, sensor arrays can be made stretchable and conformable by a number of techniques which include transferring devices onto an elastomer substrate [5], or selectively patterning and removing rigid substrates to enhance stretchability [6].

Our research develops a new approach for stretchable sensor array fabrication which simplifies the overall microelectronic fabrication process. This process utilizes excimer laser photoablation for patterning of pressure sensors and selective removal of polymer substrates to increase stretchability. Metal electrodes of the sensor and interconnects are also utilized as in-situ masks for photoablation. In this work, capacitive pressure sensor arrays are designed, fabricated, and tested as a proof of concept for this microelectronic fabrication process.

\section{FABRICATION}

Excimer laser photoablation is the process of using deep ultraviolet (DUV) radiation to pattern or etch a material directly (i.e., without requiring a post-exposure development step), and is often used for patterning of polymers. Excimer laser photoablation has been demonstrated for the fabrication of various electronics, optoelectronics, microfluidic and MEMS devices [7-9]. The threshold fluence for ablation is the lowest fluence at which the material can be effectively patterned or etched, and is known to be less than $50 \mathrm{~mJ} / \mathrm{cm}^{2}$ for many polymers [10-12]. Excimer lasers also remove thin films of metals at higher fluences [7,10]. The threshold fluence for removal or damage to metal films is a function of the metal thickness and thermal conductivity of the material [10]. Generally, since the threshold fluence for photoablation of polymers is much lower than the threshold fluence for removal or damage of high-thermally-conductive metals 
[10,11], metal thin films can be used as in-situ masks for polymers if the proper fluence is used. In addition, thermal barriers such as $\mathrm{SiO}_{2}$, placed between polymer and metal thinfilms, are effective in isolating the polymer-inorganic thinfilm interface from high temperatures experienced during photoablation and thus improve the viability of the in-situ mask [13].

The fabrication process for capacitive pressure sensors utilizes the excimer laser photoablation with in-situ masking concept with two metal layers and one sacrificial polymer layer (Fig. 1). First, the bottom metal electrode is deposited and patterned. This is followed by the deposition and photolithographic patterning of the sacrificial polymer layer. The sacrificial polymer layer will later be removed to form the structural beam and air gap. Next, a structural spin-on polymer dielectric layer will be deposited; this structural polymer dielectric layer has a low curing temperature such that the structural polymer layer can be cured without making the sacrificial layer difficult to remove. In our experiments, SU8-2000 negative cross-linking resist is used as the structural spin-on polymer due to its low temperature cross-linking characteristics. This process is followed by the deposition of a second metal layer which serves as the top electrode for the sensor array. The sample is then subjected to a flood exposure of excimer laser energy for photoablation, using the photoablation with in-situ masking concept described above. Photoablation removes all of the polymer material that is not beneath either of the metal structures. This forms patterns for sensor and interconnect structures and opens vias to remove the sacrificial polymer. The removal of the sacrificial polymer is the last step of the fabrication process.

\section{DESIGN}

MEMS-based capacitive sensors have two metal electrodes; environmental variables modify the capacitance sensed between the two electrodes. For our application, capacitive pressure sensors detect changes to pressure applied perpendicular to the plane of the substrate. The top electrode of the sensor is fabricated on top of a polymer beam or diaphragm structure, which will bend with applied pressure. The applied pressure decreases the gap between the top and bottom electrodes, which increases the sensed capacitance between the two electrodes. Sensitivity and range of the capacitive pressure sensor can be controlled by adjusting the size and width of the beam and the air gap. Individual pressure sensors are placed in an array arrangement such that each sensor can be read out by probing a unique row and column electrode. For our prototype sensor device, interconnects connecting the pressure sensors are redundant; there are two connections between each adjacent node such that the failure of one interconnect will not result in electrical failure of the sensor array. Also, interconnects are stretchable up to $30 \%$ uniaxially, such that the sensor array is conformable and can be mounted on a variety of nonplanar surfaces [13].

An analytical model is developed for the capacitive pressure sensor. There are two distinct behaviors with regards to this sensor. In the first mode of operation (Fig 2a), the beam has two boundary conditions because both ends of the (a)

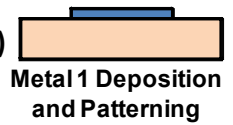

(b)

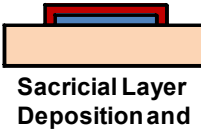
Patterning

(c)

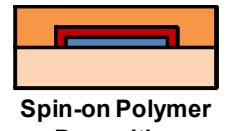

Deposition

Metal (d)

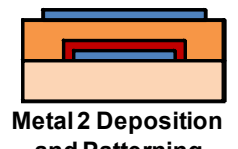

(e)

and Patterning

(f)

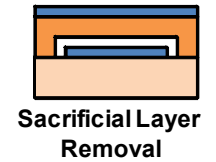

Spin-on Polymer

Sacrificial Material

Polymer Substrate

Figure 1. Cross-sectional views depicting the photoablation with in-situ masking process. (a) Deposition and patterning of metal on polymer for bottom electrode. (b) Deposition and patterning of sacrificial polymer. (c) Spin-on deposition of structural polymer. (d) Deposition and patterning of metal for top electrode. (e) Polymer photoablation process. (f) Removal of sacrificial material.

(a)

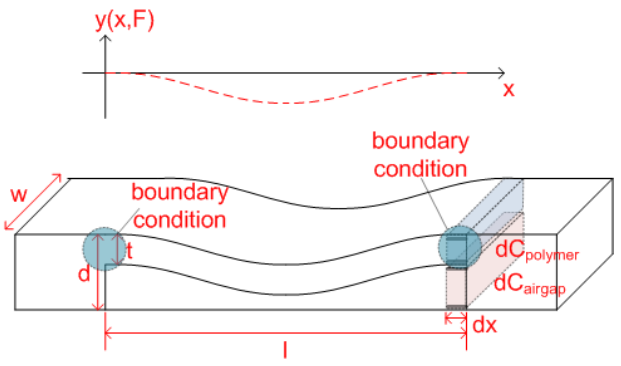

(b)

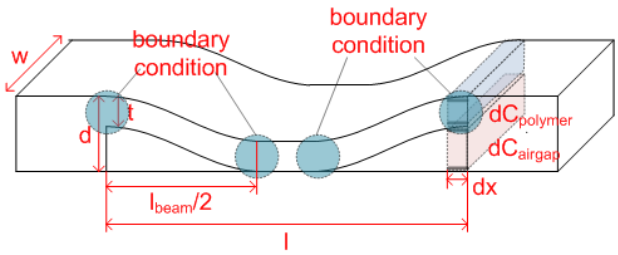

(c)

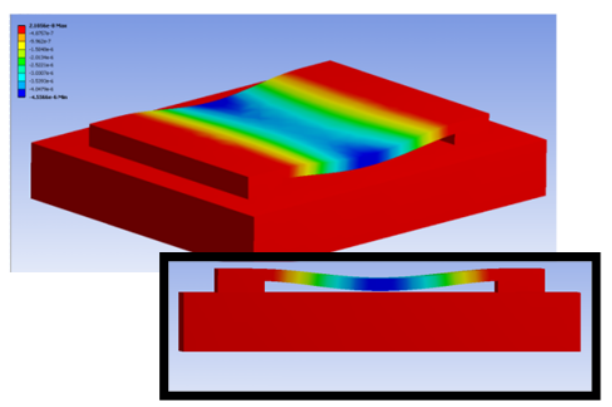

Figure 2. (a) Analytical model for Capacitive Pressure Sensor under first mode of operation. (b) Analytical model for Capacitive Pressure Sensor under second mode of operation. (c) Ansys FEA model showing deformation under applied pressure for Capacitive Pressure Sensor. 
beam are attached to the rigid structure. Equation 1 is derived from standard beam bending formulas [14], and characterizes the deformation of the beam $y$ as a function of applied force, $F$, and location $x$. $E$ and $I$ refer to the Young's modulus and Moment of Inertia of the beam, respectively.

$$
y(x, F)=-\frac{F \cdot l \cdot x^{2}}{24 \cdot E \cdot I}+\frac{F \cdot x^{3}}{12 \cdot E \cdot I}-\frac{F}{2 \cdot x^{4}}
$$

Knowing the deformation characteristics of the beam, we can find the capacitance of each small section of the beam, $d C_{\text {airgap }}$ and $d C_{\text {polymer }}$, which can be expressed as

$$
\begin{aligned}
& d C_{\text {polymer }}=\frac{\epsilon \cdot d x \cdot w}{t} \\
& d C_{\text {airgap }}=\frac{\epsilon_{0} \cdot d x \cdot w}{y(x, F)+d-t}
\end{aligned}
$$

$w$ is the width of the beam, $t$ is the thickness of the beam, $d$ is the distance between the top and bottom electrodes, $\varepsilon$ is the permittivity of the polymer, and $\varepsilon_{0}$ is the permittivity of free space. The deformation of the beam will change the size of the air gap, and thus $d C_{\text {airgap }}$, the capacitance associated with the air gap under the beam. The total capacitance is the integral sum of the series capacitances at each section of the beam, and can be expressed as

$$
C(F)=\int\left(\frac{1}{d C_{\text {airgap }}}+\frac{1}{d C_{\text {polymer }}}\right)^{-1}
$$

Equations 2, 3, and 4 can be combined and simplified to

$$
C_{\text {sensor }}(F)=\int_{0}^{l} \frac{\epsilon_{0} \cdot \epsilon \cdot w}{\epsilon \cdot(y(x, F)+d-t)+t \cdot \epsilon_{0}} d x
$$

where $l$ is the length of the beam. At higher applied pressures, the sensor enters the second mode of operation (Fig 2b), where the beam bends enough such that it is also confined in the center region. The capacitance of the sensor in the second mode of operation is

$$
\begin{aligned}
C_{\text {sensor }}(F)= & \int_{0}^{l_{\text {beam }}(F)} \frac{\epsilon_{0} \cdot \epsilon \cdot w}{\epsilon \cdot(y(x, F)+d-t)+t \cdot \epsilon_{0}} d x \\
& +\frac{\epsilon \cdot w \cdot\left(l-l_{\text {beam }}\right)}{t}
\end{aligned}
$$

where $l_{\text {beam }} / 2$ is the length of the freestanding section of the beam. Because of the additional constraints, the sensitivity of the pressure sensor is reduced in the second mode of operation. Capacitive pressure sensors are also modeled using ANSYS Finite Element Analysis (FEA) to confirm the validity of the analytical model (Fig 2c).

\section{REsults}

100um x 100um, and 200um x 200um sensor arrays are fabricated using the process described in Section II. Scanning electron micrographs (SEM) reveal that the sacrificial material is successfully removed (Fig. 3); however, the profile of the beam does not exactly resemble that of the theoretical model due to sloping of the photoresist sidewalls during the patterning of the sacrificial material. Thus, there will be some (a)

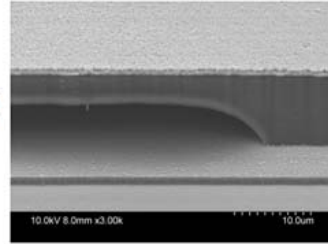

(b)

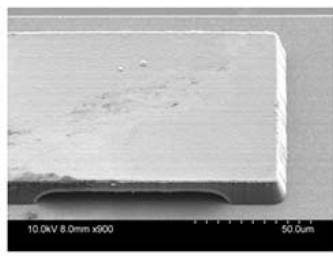

(c)

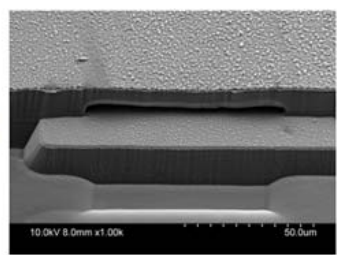

(d)

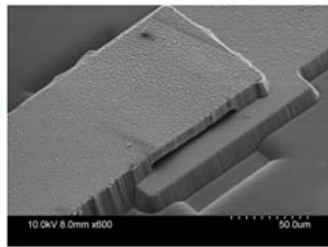

(e)

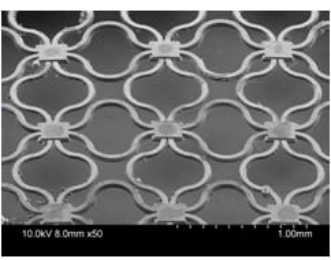

(f)

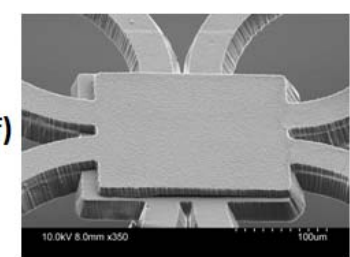

Figure 3. Scanning Electron Micrographs of capacitive pressure sensors fabricated using photoablation with in-situ masking process. (a-d):

Individual $100 \mu \mathrm{m}$ capacitive pressure sensors. (e-f): $4 \times 4200 \mu \mathrm{m}$ capacitive sensor array connected using stretchable, redundant interconnects.

deviation from the analytical model to the measured data. SEM also reveals that both electrodes of the sensor array and the corresponding layers of the interconnect structure have been successfully fabricated. As in the case of the stretchable interconnect experiments mentioned in previous work [13], conical defects are present in the experimental system, and may cause a decrease in stretchability or functionality of defects form in an inopportune location.

Individual capacitive sensors are tested and experimental results are compared with the theoretical model. The capacitance values are read out through an Analog Devices capacitance-to-digital test board, which has a resolution limit of less than $1 \mathrm{fF}$ and a range of up to $4000 \mathrm{fF}$. Parasitic capacitances are minimized using grounded coaxial cables, and any additional parasitic capacitances are accounted for in software. A micrometer stage and force meter with $5 \mathrm{mN}$ sensitivity is used to apply force in precise increments, and PDMS is used as a "buffer layer" to provide uniform pressure between the force gauge and the capacitive pressure sensor (Fig. 4), such that the applied force is distributed over the contact surface area uniformly. As predicted by the analytical models, the capacitive pressure sensor shows two distinct modes of operation, closely follows theoretical models with a beam thickness of $7.5 \mu \mathrm{m}$ to $8 \mu \mathrm{m}$, and an air gap thickness of $3 \mu \mathrm{m}$. In the first mode, the pressure sensor shows a high sensitivity, with a capacitance change of $40 \mathrm{fF}$ with an applied pressure of $150 \mathrm{kPa}$. The second mode of operation, the sensitivity is much lower; there is an additional capacitance change of $20 \mathrm{fF}$ with a total applied pressure of $500 \mathrm{kPa}$ (Fig. 5). Although the sensor response is non-linear, the dual-mode 
(a)

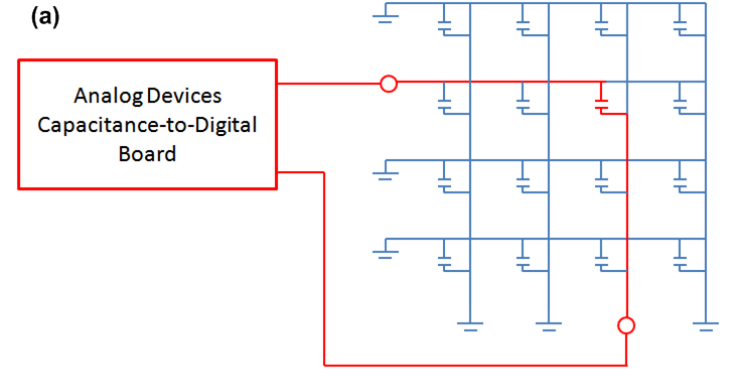

(b)

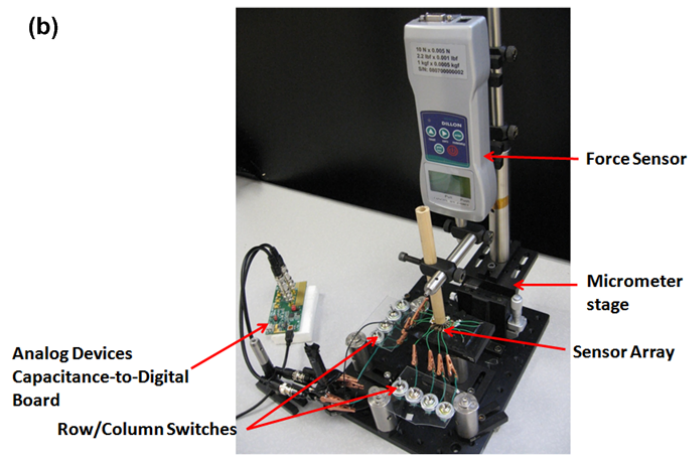

Figure 4. (a) Driving circuitry for pressure sensor array readout. The row and column of interest ( $3^{\text {rd }}$ row and $3^{\text {rd }}$ column, as shown in red) is multiplexed to the Analog Devices capacitance-to-digital board, while all other interconnects are grounded to minimize cross-talk. (b) Experimental setup for pressure sensor testing.

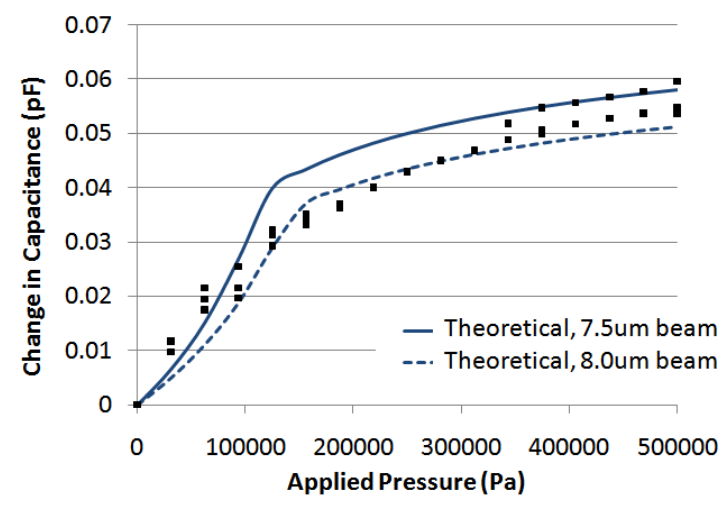

Figure 5. Experimental data of capacitive pressure sensor response to applied pressure, as compared to theoretical models with beam thicknesses of $7.5 \mu \mathrm{m}$ and $8.0 \mu \mathrm{m}$.

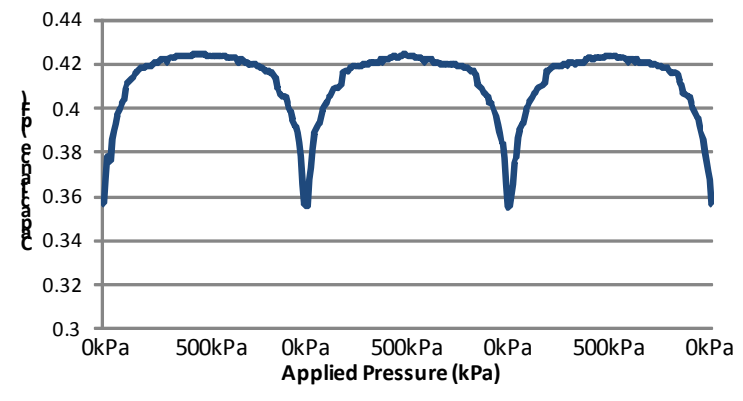

Figure 6. Sensor repeatability test. $500 \mathrm{kPa}$ of pressure is applied and released 3 time to show a repeatable response.

operation of the pressure sensor is versatile. The sensor
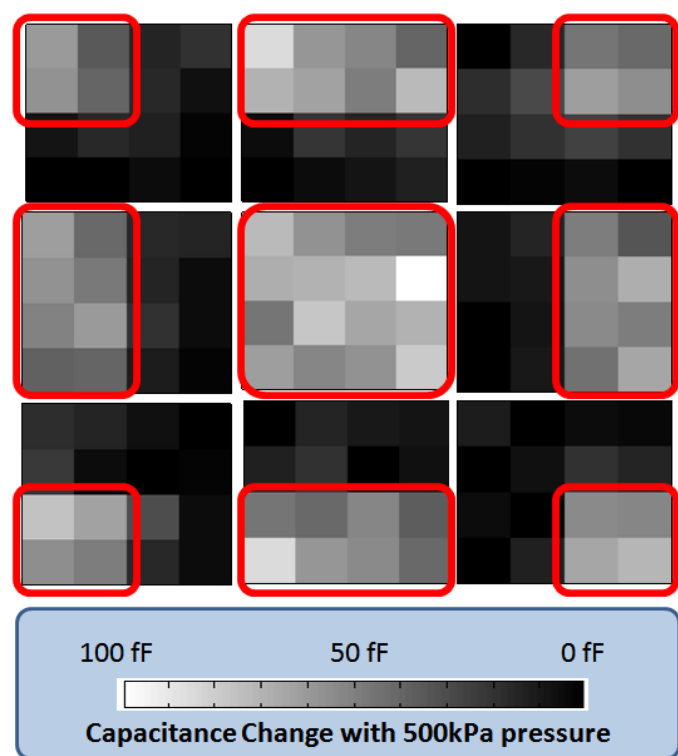

Figure 7. $4 \times 4$ Pressure Sensor Response: $500 \mathrm{kPa}$ pressure is applied to various regions of the $4 \times 4$ sensor array. Regions with applied pressure (shown in red) show an increase in capacitance.

exhibits high-sensitivity at low applied pressures but also has a large range to accommodate a wide variety of pressures. To test the repeatability of the pressure sensor, pressure is applied and released on the sensor three times, and results show a repeatable measurement over the three cycles (Fig. 6).

Capacitive sensor arrays are also tested using a similar setup (Fig. 4b). The capacitance-to-digital test board is connected to a series of switches to multiplex the signal, such that the appropriate row and column is selected to read out the sensor of interest. All other connections are grounded to minimize crosstalk between adjacent sensors. All sensors show an increase in capacitance when pressure is applied (Fig. 7); however, there is variation in the sensitivity of each sensor of the array, due to process variation. Pressure is also applied to selective regions of the sensor array (top, bottom, left, right, etc.), and the sensor array responds accordingly. That is, the regions with higher applied pressure show a correspondingly large change in capacitance, while regions with no applied pressure show only a small change in capacitance. Future experiments will include fabrication of an automated setup for sensor array testing.

\section{DISCUSSION}

The photoablation with in-situ masking process has unique advantages for the fabrication of stretchable sensor arrays. Processing is simplified through the use of excimer laser photoablation because polymer MEMS structures and interconnect meshes are patterned directly without chemical etchants. In addition, metal electrode patterns are used as insitu masks for the ablation step to eliminate one or more masking steps. Furthermore, all fabrication steps are batch processes which can be scaled up to large-area design. Sensor and interconnect patterns are also defined lithographically, so that the full fabrication process sequence is compatible with a wide variety of designs. 
Future experiments will focus on process optimizations to reduce the variations of sensor response between different elements of the array. Sensor arrays can also be fabricated in a large array of capacitive pressure sensors using large-area fabrication technique demonstrated with interconnects in previous work [13]. Also, the applicability of this fabrication process can be extended to other capacitive MEMS-based sensors such as shear sensors, accelerometers, and condenser microphones, since all capacitive sensors contain two metal electrodes and thus are compatible with the photoablation with in-situ masking process.

\section{CONCLUSION}

Stretchable sensor arrays enable sensors to be mounted on non-planar surfaces, such as the skin of a robotic hand, giving additional feedback for robotic systems which is vital for interactions with fragile objects or humans. Our research focuses on developing a unique fabrication process photoablation with in-situ masking concept - which uses metal patterns serve both as functional electrode layers and as in-situ masks for excimer laser photoablation. This approach reduces the number of photomasks and processing steps, is scalable for large-area arrays, and is adaptable for a variety of materials and designs. Individual $200 \mu \mathrm{m}$-sized sensors fabricated using this approach show a capacitance change of $40 \mathrm{fF}$ with an applied pressure of $150 \mathrm{kPa}$, and a capacitance change of $60 \mathrm{fF}$ with an applied pressure of $500 \mathrm{kPa}$. Pressure sensors are fabricated as a $4 \times 4$ array connected with redundant stretchable interconnects to demonstrate successful readout of different pressure profiles. These results are the cornerstone for future experiments which will investigate larger capacitive pressure sensor arrays. Furthermore, different modalities of capacitive MEMS-based sensors fabricated using a similar fabrication process.

\section{REFERENCES}

[1] S.-P. Chang, M. G. Allen, "Demonstration for integrating capacitive pressure sensors with read-out circuitry on stainless steel substrate," Sensors and Actuators A, vol. 116, pp. 195-204, 2004.

[2] T. Someya, T. Sekitani, S. Iba, Y. Kato, H. Kawaguchi, T. Sakurai, "A large-area, flexible pressure sensor matrix with organic field-effect transistors for artificial skin applications," Proc. National Academy of Sciences, vol. 101, pp. 9966-9970, 2004.

[3] T. Someya, Y. Kato, T. Sekitani, S. Iba, Y. Noguchi, Y. Murase, H. Kawaguchi, T. Sakurai, "Conformable, flexible, large-area networks of pressure and thermal sensors with organic transistor active matrixes," Proc. National Academy of Sciences, vol. 102, pp. 12321-12325, 2005.

[4] J. Engel, J. Chen, Z. Fan, C. Liu, "Polymer micromachined multimodal tactile sensors," Sensors and Actuators A, vol. 117, pp. 50-61, 2005.

[5] D.-H. Kim, J. A. Rogers, "Stretchable electronics: materials strategies and devices," Advanced Materials, vol. 20, pp. 4487-4892, 2008.

[6] K. Huang, P. Peumans, "Stretchable silicon sensor networks for structural health monitoring," Proc. SPIE, vol. 6174, pp. 617412-1-10, 2006

[7] K. Jain, Excimer Laser Lithography, Bellingham, WA: SPIE Optical Engineering Press, 1990.

[8] M. Wehner, "Ablative micro-fabrication," in Excimer Laser Technology, D. Basting and G. Marowsky, eds., Berlin: SpingerVerlag, 2005, pp. 149-154.

[9] R. Srinivasan, B. Braren, R. W. Dreyfus, "Ultraviolet laser ablation of polyimide films," Journal of Applied Physics, vol. 61, p. 372, Jan. 1987.

[10] J. Siegel, K. Ettrich, E. Welsch, E. Matthais, "UV-laser ablation of ductile nabrittle metal films," Applied Physics A, vol. 64, pp. 213-218, Feb. 1997.

[11] J. Chae, K. Jain, "Excimer laser projection photoablation patterning of metal thin films for fabrication of microelectronic devices and displays," 2008 IEEE Photonics Technology Letters, vol. 20, pp. 1216$1218,2008$.

[12] M. Gonzalez, F. Axisa, M. V. Bulcke, D. Brosteaux, B. Vandevelde, J. Vanfleteren, "Design of metal interconnects for stretchable electronic circuits using finite element analysis," Proc. Thermal, Mechanical, and Multi-Phyiscs Simulation Experiments in Microelectronics and MicroSystems, pp. 1-6, Apr. 2007.

[13] K. Lin, J. Chae, K. Jain, "Design and fabrication of large-area, redundant, stretchable interconnect meshes using excimer laser photoablation and in-situ masking" IEEE Transactions on Advanced Packaging, in press.

[14] W. C. Young, Roark's Guide to Stress and Strain, $6^{\text {th }}$ ed., New York: McGraw-Hill Book Company, 1989, pp. 100-103. 